\title{
Protective Effects of Exercise and Insulin Against Stress-Induced Impairment of Spatial Learning
}

\author{
Ahmad Rahmani $i^{1,{ }^{*}}$ and Naser Naghdi ${ }^{2}$ \\ ${ }^{1}$ Department of Sport Sciences, Faculty of Humanities, University of Zanjan, Zanjan, Iran \\ ${ }^{2}$ Department of Physiology, Pasteur Institute of Iran, Tehran, Iran \\ "Corresponding author: Department of Sport Sciences, Faculty of Humanities, University of Zanjan, Zanjan, Iran. Email: a_rahmani@znu.ac.ir \\ Received 2018 January 25; Revised 2018 May 02; Accepted 2018 September 23.
}

\begin{abstract}
Background: Studies have shown that exercise and insulin can improve cognitive function. However, little is known about the combined effects of insulin and exercise on memory and learning under stress conditions.

Objectives: This study was designed to investigate the effects of peripheral insulin injection and/or physical exercise on stressinduced memory deficit.

Methods: Adult male Wistar rats (200 - $220 \mathrm{~g})$ received immobilization stress $(2 \mathrm{~h} / \mathrm{d} \times 7 \mathrm{~d})$, mild-intensity exercise $(30 \mathrm{~min} / \mathrm{d} \times 7 \mathrm{~d})$, intraperitoneal (i.p.) injection of insulin $(1,6,12 \mathrm{IU} / \mathrm{kg})$ or saline, insulin-exercise treatment, or no intervention. The animals were assigned on a random basis to 21 experimental groups $(\mathrm{N}=10)$. They were trained and tested in the Morris water maze.

Results: Chronic restraint stress impaired spatial performance in the Morris water maze $(\mathrm{P}<0.05)$ and elevated serum corticosterone levels $(\mathrm{P}<0.001)$. Detrimental effects of stress were alleviated by exercise $(\mathrm{P}<0.01)$. The lower dose $(1 \mathrm{IU} / \mathrm{kg})$ of insulin protected the animals against the deleterious effects of stress. The combined effects of exercise and insulin (low and moderate doses) prevented stress-induced learning and memory impairment.

Conclusions: Insulin and/or exercise can prevent stress-induced memory deficits. The cognitive benefits of exercise continue to evolve after cessation of treatment. Insulin-exercise treatment is as effective as exercise alone and insulin alone for preventing the detrimental effects of stress.
\end{abstract}

Keywords: Insulin, Exercise, Spatial Memory, Neuroprotection, Corticosterone

\section{Background}

Under stress conditions the human body secretes glucocorticoids. In rats, corticosterone is the major glucocorticoid involved in energy regulation, immune responses, and stress reactions. Secretion of this hormone allows a fight-or-flight action by activating the endogenous substrate and motivating a state of insulin resistance in the liver and skeletal muscles.

Chronic stress has damaging effects on the brain and especially affects the hippocampal structure and function leading to cognitive and mood disturbances (1). The hippocampus is an important structure for spatial learning and memory, which is enriched with corticosteroid receptors (2). This means that it is susceptible to stressinduced corticosteroid impairment (3). Stress can induce dendritic retraction in the hippocampal CA3 subregion following prolonged restraint (4-6) or chronic social stress (7). Chronic stress can also increase apoptosis in the hippocampus, thereby causing memory decline (8). Five hours of restraint stress has been shown to produce modest impairment in a novel object recognition task (9). Importantly, these stress-induced cognitive disturbances are likely to be exaggerated in the absence of physical activity (1).

Kim and Seo exposed rats to electric shock stress (seven consecutive days) and treadmill exercise running (30 $\mathrm{min} / \mathrm{d} \times 4 \mathrm{~d}$ ); they showed that exercise increases neurogenesis, inhibits apoptosis, and relieves stress-induced destruction of spatial learning (10). Treadmill running can also alleviate repeated stress-induced spatial memory deterioration by removing reactive oxygen species (ROS) in the hippocampus and activation of brain-derived neurotrophic factor (BDNF) signaling (11). Moreover, physical exercise prevents memory loss and leads to reduced insulin resistance (12). However, little is known about the concurrent effects of exercise and insulin treatments on memory performance.

The high density of insulin/insulin receptors in the brain areas such as the hippocampus and cerebral cortex 
plays a critical role in higher cognitive function. This indicates that insulin could be involved in the modification of memory (13). Also, it promotes neuroplasticity in the developing and adult brain (14). Accordingly, after water maze training, gene expression of insulin receptors shows upregulation in the CA1 region (15). Some reports present that insulin prevents memory loss. For example, Moosavi et al. demonstrated that intrahippocampal insulin injection protected against stress-induced memory impairment (3). Similarly, one week of intranasal administration of insulin prevented anesthesia-induced spatial learning and memory deficit(16). On the other hand, other studies report that intraperitoneal (IP) administration of insulin can impair spatial memory in a dose-dependent manner $(17,18)$.

Moreover, insulin seems to have neuroprotective effects against memory impairment induced by stress, drugs, and diseases $(3,16,19)$. The neuroprotective mechanisms of insulin include the regulation of neurotransmitters, promotion of glycogen synthesis, and inhibition of neuronal necrosis and apoptosis (20).

Literature review shows that similar studies have examined the effect of insulin on spatial learning and memory and have produced contradictory results. In addition, there is no evidence as to the effect of systemic insulin administration alone or in combination with exercise on cognitive function in stressed and non-stressed animals. Therefore, this study was designed to examine the effect of i.p. insulin administration and/or exercise on spatial learning and memory under immobilization stress and normal conditions.

\section{Methods}

\subsection{Animals}

Male Wistar rats (200 - $220 \mathrm{~g}$ ) were purchased from Pasteur Institute of Iran. They were placed on a 12/12-h lightdark circuit in a temperature $\left(25 \pm 2^{\circ} \mathrm{C}\right)$ and humidity (\%45 \pm 5 ) controlled room. Food and water were provided ad libitum. All experimental procedures were in accordance with the National Institutes of Health (NIH) Guide for the Care and Use of Laboratory Animals. After one week of acclimation, the animals were randomly assigned into 21 groups of 10 (Figure 1).

\subsection{Drugs}

Insulin was provided from Exir Pharmaceutical Company (Borujerd, Iran) and diluted in sterile $0.9 \%$ saline. It was administered intraperitoneally at three different doses $(1,6$, and $12 \mathrm{IU} / \mathrm{kg})$. Also, normal saline was injected at the same volume as a vehicle to the saline control groups. Drugs were administered once a day for seven sequential days at 10:00 - 10:30 a.m.

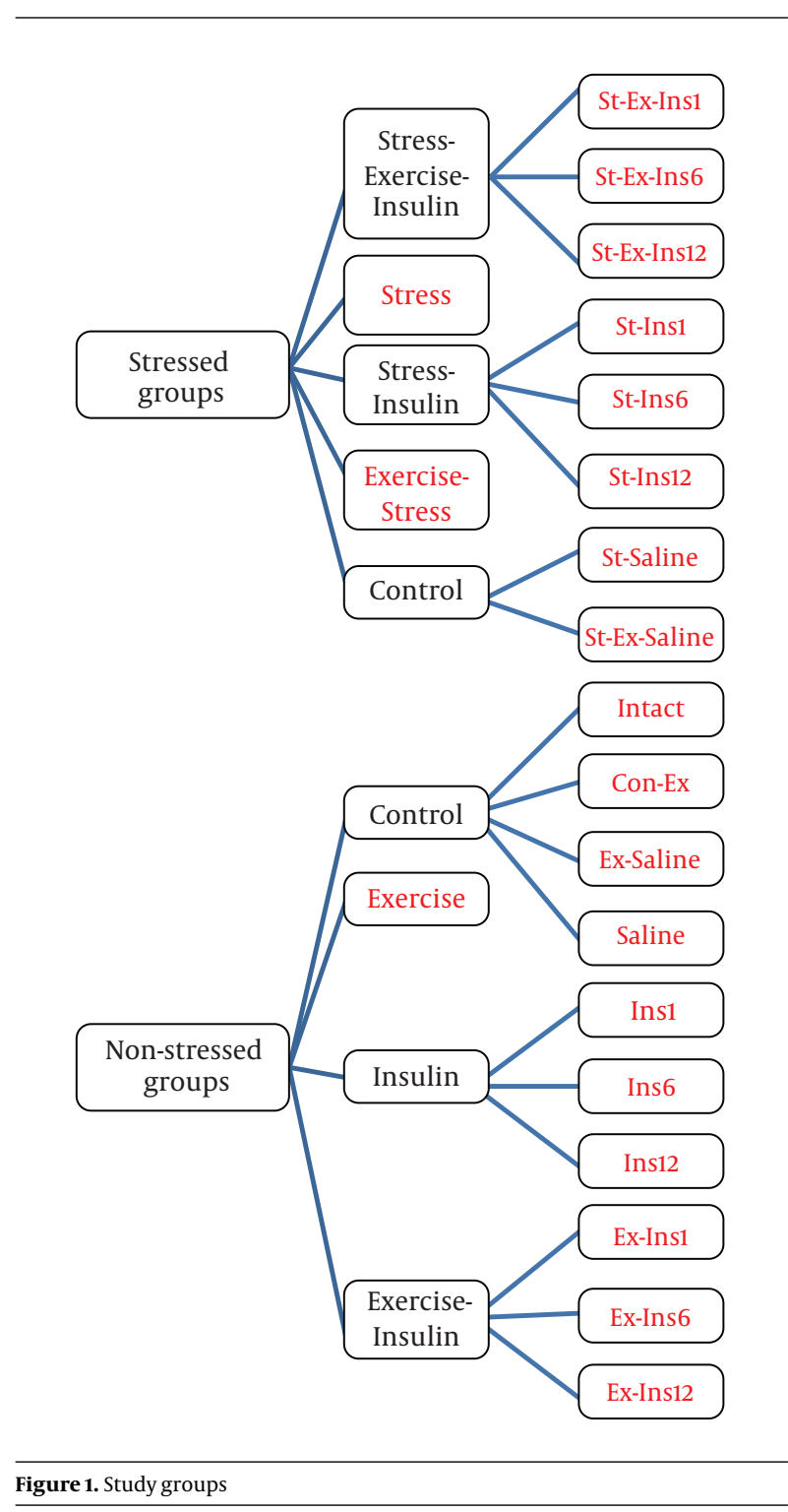

\subsection{Immobilization Stress, Corticosterone and Glucose Assay}

Animals were immobilized in a plexiglass rats restrainer and were placed in the back recumbent position for $2 \mathrm{~h} / \mathrm{d}$ for 7 days, at 11:00 - 13:00 (3). Evaluation of the effectiveness of immobilization stress was assessed by the physical symptoms of stress, such as body tremors, teeth gnashing, urination, and yawning. At the end of the seventh day of stress induction, after light ether anesthesia, blood samples from all the study groups were taken directly from the heart. Serum corticosterone and glucose levels were assessed using a commercial rat corticosterone ELISA kit (Marburg, Germany) and Glucose Colorimetric Enzymatic kit (Parsazmun, Iran). 


\subsection{Exercise Protocol}

The animals in the exercise group underwent 30minutes treadmill running. The load for exercise groups included treadmill training at a speed of $4 \mathrm{~m} / \mathrm{min}$ for 10 minutes. This was followed by running for 10 minutes at a speed of $7 \mathrm{~m} / \mathrm{min}$ and ended with 10 minutes running at 10 $\mathrm{m} / \mathrm{min}$ at 0 degrees of inclination. Running was conducted once daily at 7:00 - 8:00 a.m. for seven consecutive days. Low level of exercise intensity was used to eliminate any other stressful conditions that may produce physical and physiological alterations in the laboratory animals (21).

\subsection{Behavioral Procedure}

Two days after completion of the stress induction, the animals were trained in the Morris water maze. The acquisition phase consisted of one block of four trials per day for four days. Water maze training consisted of placing the rat into the water facing the wall of the tank at each of the four starting points and allowing it to swim and locate the hidden platform. Starting points were different in a quasirandom manner, such that each trial was begun once in each location.

The task needs rats to swim to the hidden platform using periphery spatial cues. After finding the platform, the animals were allowed to stay there for 30 seconds before the start of the next trial. If the rat failed to find the platform during 60 seconds, it was placed on the platform for 30 seconds. Latency to the platform and the swimming distance was recorded. On the 10th day, we repeated the test (recall/retrieval phase); each animal performed one block of four trials as had executed in the first four days. Following 60 minutes, the visible test was conducted. It evaluates animals' motivation and vision. Performance in the Morris water maze was recorded using EthoVision XT-Noldus video tracking system. Figure 2 illustrates various steps in the study.

\subsection{Statistical Analysis}

After ensuring about the assumptions of normality (Shapiro-Wilk test) and homogeneity of variance (Levene's test), data were analyzed using Split-Plot Analysis of Variance (SPANOVA) and LSD post hoc test. The effects of exercise and stress treatments on corticosterone levels were assessed using ANOVA followed by planned comparisons. P value less than 0.05 was considered significant. SPSS version 16.0 was used for statistical analysis. Diagrams were drawn using Microsoft Office Excel 2010.

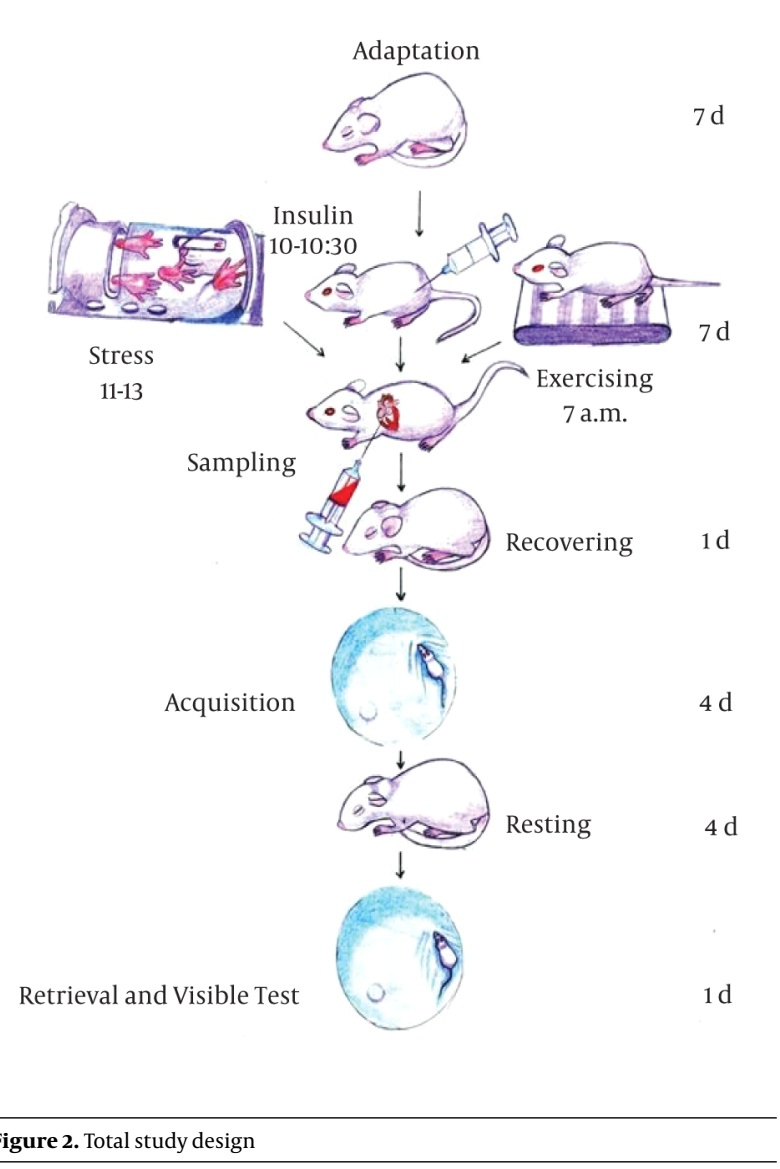

\section{Results}

3.1. Effects of Insulin or Exercise Treatment on Corticosterone Levels

ANOVA showed significant differences in serum corticosterone levels between the groups $\left(\mathrm{F}_{(3,16)}=82.79, \mathrm{P}<\right.$ $0.001, \eta^{2}=0.94$; Figure 3$)$. The planned comparison revealed that stressed rats had significantly higher levels of corticosterone than the control group $\left(t_{(4.26)}=-6.65, \mathrm{P}=\right.$ 0.002). On the other hand, exercise ameliorated stressinduced elevation of serum corticosterone $\left(t_{(8.08)}=70.74\right.$, $\mathrm{P}<0.001$; Figure 3). However, administration of insulin at all the doses (i.e., 1, 6, and $12 \mathrm{IU}$ ) did not have any effects on corticosterone level.

\subsection{Effect of Immobilization Stress on Learning and Memory}

Chronic immobilization stress impaired learning and memory. Stressed animals had a higher escape latency $\left(\mathrm{F}_{(1,18)}=7.45, \mathrm{P}=0.014, \eta^{2}=0.29\right)$ and distance traveled $\left(\mathrm{F}_{(1,18)}\right.$ $\left.=7.58, \mathrm{P}=0.013, \eta^{2}=0.3\right)$ than the control group. Also, there was a significant difference in retrieval phase $\left(\mathrm{F}_{(1,18)}=4.86\right.$, $\left.\mathrm{P}=041, \eta^{2}=0.21\right)$ (data not shown). 


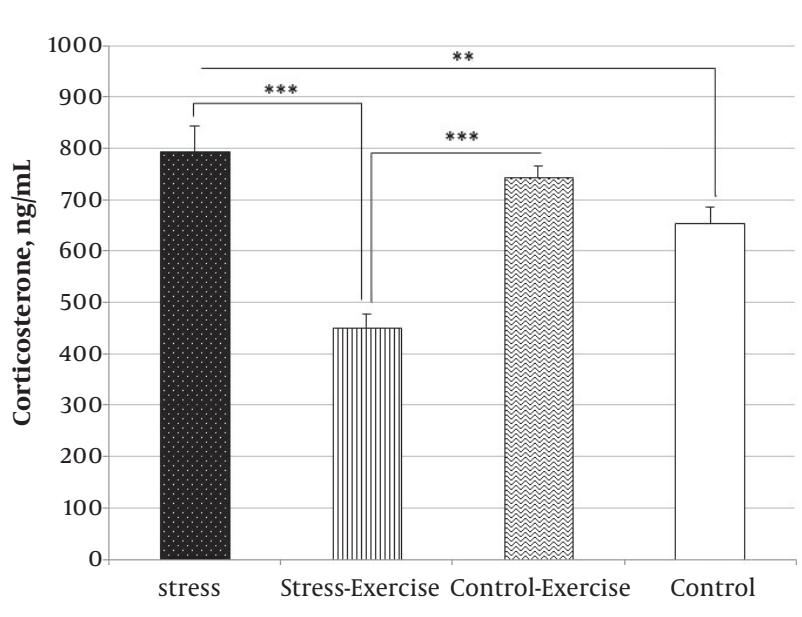

Figure 3. The effects of stress and exercise treatment on serum corticosterone level immediately following immobilization (13:00 p.m) on the 7th day of stress treatment. ${ }^{* * *}$ indicates $\mathrm{P}<0.001$ and ${ }^{* *}$ indicates $\mathrm{P}<0.01$.

\subsection{Protective Effect of Exercise}

Escape latency $(\mathrm{P}=0.023)$ and distance traveled $(\mathrm{P}=$ $0.002)$ in the animals treated with stress-exercise were better than those in the stress group (Figure 4). There were no significant differences among the groups in recall test (data not shown).

Moreover, exercise in non-stress conditions improved spatial memory retrieval compared to the control group (data not shown).

\subsection{Protective Effect of Insulin}

Animals treated with the lower dose of insulin (1 IU insulin-stress) demonstrated better spatial learning than the stress-saline group, as latency to the platform ( $\mathrm{P}=$ $0.008)$ and distance traveled $(\mathrm{P}=0.014)$ were significantly better (Figures $5 \mathrm{~A}$ and $5 \mathrm{~B})$. ANOVA revealed significant differences among these groups $\left(\mathrm{F}_{(3,32)}=3.95, \mathrm{P}=0.017\right)$ in the retrieval phase. The average dose of insulin (6 IU) did not alleviate stress-induced memory impairment (Figure 5C).

Moreover, insulin in non-stress conditions improved learning and memory in a dose dependent manner (data not shown). There were no significant differences in serum glucose levels among the groups; therefore, the water maze performance has not been affected by glucose levels (data not shown).

\subsection{Protective Effect of Exercise-Insulin}

There were significant differences among interactive groups in escape latency $\left(\mathrm{F}_{(4,44)}=3.81, \mathrm{P}=0.01, \eta^{2}=0.26\right)$ and distance traveled $\left(\mathrm{F}_{(4,44)}=4.5, \mathrm{P}=0.004, \eta^{2}=0.29\right)$. Animals in ex-do-st (exercise-saline-stress), ex-d1-st (exerciseinsulin dose 1-stress), and ex-d6-st (exercise-insulin dose 6stress) groups demonstrated better spatial learning than stress-saline treated animals. Rats treated with ex-d12-st (exercise-insulin dose12-stress) showed no significant differences compared with the st-do (stress-saline) treated group. The results of the recall test revealed no significant differences among the groups $\left(\mathrm{F}_{(4,44)}=1.21, \mathrm{P}=0.32\right)$.

In all the study groups, the results of swimming speed and visible test showed no significant differences compared with the control groups.

\section{Discussion}

\subsection{Effect of Stress on Learning and Memory}

Immobilization stress significantly impaired performance in both acquisition and retrieval phases. Evidence suggests that chronic stress and exposure to corticosterone can cause dendritic atrophy and nerve damage (22). It also decreases synapse formation in the hippocampus (23). Such changes in the hippocampus have been presented as the underlying mechanisms for stress-induced cognitive impairment, and generally, they are attributed to changes in corticosterone levels.

\subsection{Effect of Exercise on Stressed Subjects}

Exercise alleviated stress-induced impairment in the acquisition phase. This is consistent with the results of Kim et al. (24). They reported that treadmill running relieved short-term memory loss by increasing cell proliferation and suppression of apoptosis in the hippocampal dentate gyrus (24). Stress increases glutamate and its neurotoxic effects (8), whereas exercise can up-regulate glutamate transporters (EAAC1), which are responsible for the elimination of glutamate within the synaptic gap. Moreover, glutamate receptors that mediate glutamate neurotoxic effects can be down-regulated by exercise (15).

Furthermore, results revealed that exercise significantly decreased corticosterone levels in stressed (stressexercise) animals. Regular exercise provides opportunities for an organism to react to changes in environmental conditions by means of physiological modifications such as metabolic rate, mitochondrial biogenesis and hypothalamic-pituitary-adrenal (HPA) adaptability (15). It seems that normal functioning of spatial learning and memory, which are compromised by immobilization stress, may be affected by physical activity.

In addition, results showed that in non-stress condition exercise has delayed effect. Although exercise had no significant effect in the acquisition phase, it improved 

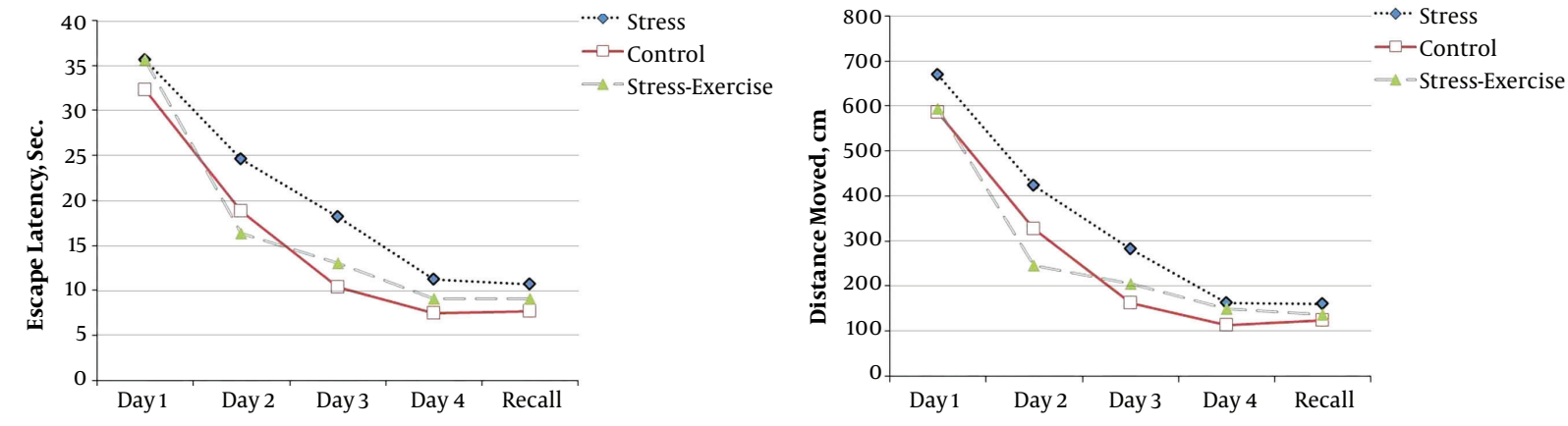

Figure 4. The effect of exercise on learning and memory following stress intervention. A, Average escape latency; B, average distance traveled.

A

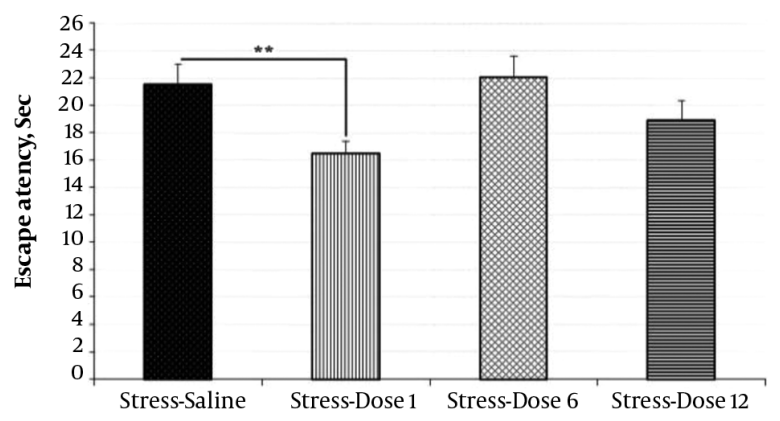

B

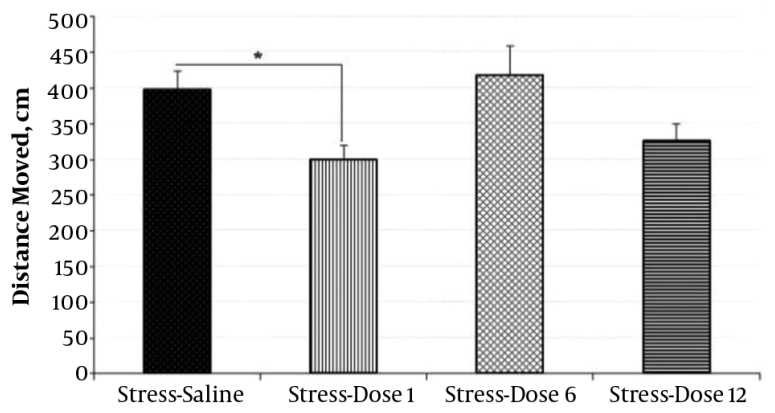

C

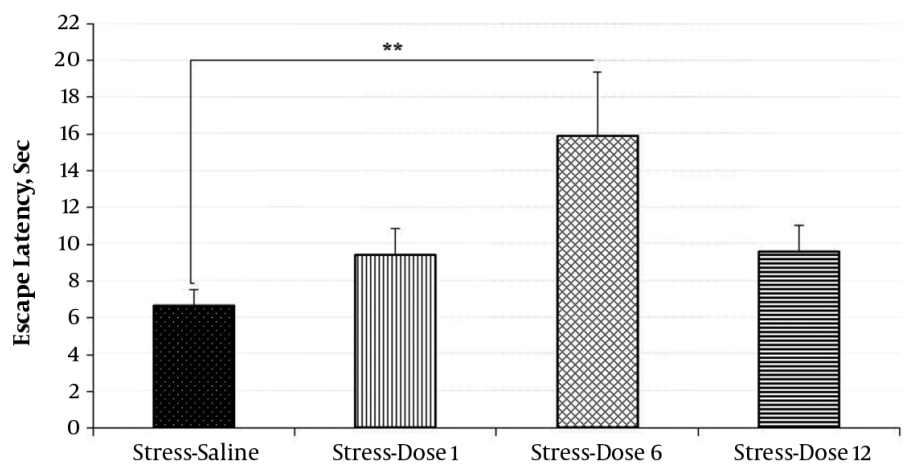

Figure 5. The effect of insulin administration on learning and memory in stressed animals. A, Average escape latency; B, average distance traveled; $C$, the recall test. * and ${ }^{* *}$ indicate $\mathrm{P}<0.05$ and $\mathrm{P}<0.01$, respectively.

memory in the retention stage. This is consistent with the results of Darkhah et al. (25). Plasticity-related responses of genes have different temporal patterns of gene induction in response to exercise (26). Therefore, it seems that the effects of exercise are more long-lasting than previously thought (27).

\subsection{Effect of Insulin on Stressed Subjects}

Although insulin had no effects on stress-induced corticosterone release, it alleviated the detrimental effects of stress during the acquisition phase at the low dose (1 IU). It seems that low-dose insulin administration has an improving effect on spatial memory. Consistent with this result, Reger et al. showed that following intravenous infusion 
of insulin, memory improved at only very low doses (28). Dose-dependent responses may be due to the amount of insulin carried to the central nervous system after the systemic administration (28). Future studies should explore these possibilities.

It appears that changes in insulin are independent of changes in serum corticosterone. Likewise, spatial learning, which is compromised by restraint stress, may be dependent on insulin for normal functioning.

Studies have revealed that psychological stress in human and immobilization stress in lab animals increases oxidative stress and apoptosis (29). Oxidative stress decreases the uptake of gamma-aminobutyric acid (GABA) and glutamate and increases extrasynaptosomal glutamate levels. Insulin treatment reverses the decrease in GABA accumulation. Thus, recovery of neuronal performance is one mechanism whereby insulin can protect neurons against stress (30).

In the present study, despite the protective effect of insulin in the acquisition phase, it had no effects in the retrieval stage. It should be noted that the time interval between the last insulin treatment and retrieval test was nine days. It is postulated that during this period the effect of insulin could have decreased; hence, animals' performance had returned to levels of the control group.

\subsection{Combination Effect of Exercise and Insulin on Stressed Sub- jects}

Our results demonstrated that the interactive effects of exercise and insulin can modulate the degenerative effects of stress. Exercise significantly improves peripheral insulin sensitivity and spatial learning (31). Therefore, insulin intake when its signaling has already increased, for example following exercise, could improve cognitive performance in a dose-dependent manner.

However, the body of research in this area has several limitations. First, the current method to induce stress is not common in real-life situations due to the technical and ethical concerns. Also, in this study, cellular changes such as hippocampal neurogenesis and apoptosis were not assessed; thus, the mechanisms underlying the effects of the treatments remain unclear. Moreover, the length of the exercise regimens may not be long enough to make appropriate changes. Clinical studies have shown that different kinds of exercise, including aerobic, resistance, and dance, can improve cognitive function, even though the appropriate type, quantity, mechanisms, and duration of exercise are unclear (32).

\subsection{Conclusion}

The present study provides evidence that restraint stress could produce physical, physiological, and cognitive effects. Low-intensity regular exercise and/or peripheral insulin administration counteracts the effects of stress and causes behavioral adaptation in spite of the activation of the HPA axis. In addition, concurrent administration of exercise and insulin can counteract the negative effects of stress on learning in a dose-dependent manner, and exercise alone (exercise-stress) has the same effect. Ultimately, exercise benefits continue even after a delay between exercise and cognitive test.

\section{Footnote}

Ethical Considerations: Ethical code: Znu.ECRA. 2018-2.

\section{References}

1. Tsatsoulis A, Fountoulakis S. The protective role of exercise on stress system dysregulation and comorbidities. Ann N Y Acad Sci. 2006;1083:196-213. doi: 10.1196/annals.1367.020. [PubMed: 17148741].

2. Rendeiro C, Spencer JP, Vauzour D, Butler LT, Ellis JA, Williams CM. The impact of flavonoids on spatial memory in rodents: From behaviour to underlying hippocampal mechanisms. Genes Nutr. 2009;4(4):25170. doi: 10.1007/s12263-009-0137-2. [PubMed: 19727888]. [PubMed Central: PMC2775889].

3. Moosavi M, Naghdi N, Maghsoudi N, Zahedi Asl S. Insulin protects against stress-induced impairments in water maze performance. Behav Brain Res. 2007;176(2):230-6. doi: 10.1016/j.bbr.2006.10.011. [PubMed: 17116337].

4. Christian KM, Miracle AD, Wellman CL, Nakazawa K. Chronic stress-induced hippocampal dendritic retraction requires CA3 NMDA receptors. Neuroscience. 2011;174:26-36. doi: 10.1016/j.neuroscience.2010.11.033. [PubMed: 21108993]. [PubMed Central: PMC3020251].

5. Leuner B, Shors TJ. Stress, anxiety, and dendritic spines: What are the connections? Neuroscience. 2013;251:108-19. doi 10.1016/j.neuroscience.2012.04.021. [PubMed: 22522470].

6. Conrad CD. What is the functional significance of chronic stressinduced CA3 dendritic retraction within the hippocampus? Behav Cogn Neurosci Rev. 2006;5(1):41-60. doi: 10.1177/1534582306289043. [PubMed: 16816092]. [PubMed Central: PMC1512384].

7. Wang XD, Chen Y, Wolf M, Wagner KV, Liebl C, Scharf SH, et al. Forebrain CRHR1 deficiency attenuates chronic stress-induced cognitive deficits and dendritic remodeling. Neurobiol Dis. 2011;42(3):300-10. doi: 10.1016/j.nbd.2011.01.020. [PubMed: 21296667]. [PubMed Central: PMC3200197].

8. Sung YH, Shin MS, Cho S, Baik HH, Jin BK, Chang HK, et al. Depressionlike state in maternal rats induced by repeated separation of pups is accompanied by a decrease of cell proliferation and an increase of apoptosis in the hippocampus. Neurosci Lett. 2010;470(1):86-90. doi: 10.1016/j.neulet.2009.12.063. [PubMed: 20043974].

9. Maras PM, Molet J, Chen Y, Rice C, Ji SG, Solodkin A, et al. Preferential loss of dorsal-hippocampus synapses underlies memory impairments provoked by short, multimodal stress. Mol Psychiatry. 2014;19(7):811-22. doi: 10.1038/mp.2014.12. [PubMed: 24589888]. [PubMed Central: PMC4074447].

10. Kim BK, Seo JH. Treadmill exercise alleviates post-traumatic stress disorder-induced impairment of spatial learning memory in rats. J Exerc Rehabil. 2013;9(4):413-9. doi: 10.12965/jer.130058. [PubMed: 24278894]. [PubMed Central: PMC3836542]. 
11. Kang JS. Exercise copes with prolonged stress-induced impairment of spatial memory performance by endoplasmic reticulum stress. J Exerc Nutrition Biochem. 2015;19(3):191-7. doi: 10.5717/jenb.2015.15080705. [PubMed: 26527209]. [PubMed Central: PMC4624120].

12. Park HS, Cho HS, Kim TW. Physical exercise promotes memory capability by enhancing hippocampal mitochondrial functions and inhibiting apoptosis in obesity-induced insulin resistance by high fat diet. Metab Brain Dis. 2018;33(1):283-92. doi: 10.1007/s11011-017-0160-8. [PubMed: 29185193].

13. Haj-ali V, Mohaddes G, Babri SH. Intracerebroventricular insulin improves spatial learning and memory in male Wistar rats. Behav Neurosci. 2009;123(6):1309-14. doi: 10.1037/a0017722. [PubMed: 20001114].

14. Ferrario CR, Reagan LP. Insulin-mediated synaptic plasticity in the CNS: Anatomical, functional and temporal contexts. Neuropharmacology. 2018;136(Pt B):182-91. doi: 10.1016/j.neuropharm.2017.12.001. [PubMed: 29217283]. [PubMed Central: PMC5988909].

15. Dou JT, Chen M, Dufour F, Alkon DL, Zhao WQ. Insulin receptor sig naling in long-term memory consolidation following spatial learning. Learn Mem. 2005;12(6):646-55. doi: 10.1101/lm.88005. [PubMed 16287721]. [PubMed Central: PMC1356184].

16. Zhang Y, Dai CL, Chen Y, Iqbal K, Liu F, Gong CX. Intranasal in sulin prevents anesthesia-induced spatial learning and memory deficit in mice. Sci Rep. 2016;6:21186. doi: 10.1038/srep21186. [PubMed: 26879001]. [PubMed Central: PMC4754754].

17. Akanmu MA, Nwabudike NL, Ilesanmi OR. Analgesic, learning and memory and anxiolytic effects of insulin in mice. Behav Brain Res. 2009;196(2):237-41. doi: 10.1016/j.bbr.2008.09.008. [PubMed 18840474].

18. Kopf SR, Baratti CM. The impairment of retention induced by insulin in mice may be mediated by a reduction in central cholinergic activity. Neurobiol Learn Mem. 1995;63(3):220-8. doi: 10.1006/nlme.1995.1026. [PubMed: 7670835].

19. Holscher C. First clinical data of the neuroprotective effects of nasal insulin application in patients with Alzheimer's disease. Alzheimers Dement. 2014;10(1 Suppl):S33-7. doi: 10.1016/j.jalz.2013.12.006 [PubMed: 24529523].

20. Yu LY, Pei Y. Insulin neuroprotection and the mechanisms. Chin Med $J$ (Engl). 2015;128(7):976-81. doi: 10.4103/0366-6999.154323. [PubMed: 25836621]. [PubMed Central: PMC4834017].

21. Kim SH, Kim HB, Jang MH, Lim BV, Kim YJ, Kim YP, et al. Treadmill exercise increases cell proliferation without altering of apoptosis in dentate gyrus of Sprague-Dawley rats. Life Sci. 2002;71(11):1331-40. doi: 10.1016/S0024-3205(02)01849-0. [PubMed: 12106598].

22. Reisi P, Alaei H, Babri S, Sharifi MR, Mohaddes G. Effects of treadmill running on spatial learning and memory in streptozotocin- induced diabetic rats. Neurosci Lett. 2009;455(2):79-83. doi: 10.1016/j.neulet.2009.03.052. [PubMed: 19368850].

23. McEwen BS, Rasgon NL. The brain and body on stress allostatic load and mechanisms for depression and dementia. Depression As a Systemic Illness. 2018. $14 \mathrm{p}$.

24. Kim YH, Sung YH, Lee HH, Ko IG, Kim SE, Shin MS, et al. Postnatal treadmill exercise alleviates short-term memory impairment by enhancing cell proliferation and suppressing apoptosis in the hippocampus of rat pups born to diabetic rats. J Exerc Rehabil. 2014;10(4):20917. doi: 10.12965/jer.140145. [PubMed: 25210695]. [PubMed Central: PMC4157927].

25. Darkhah P, Zarghami M, Shetab Bushehri N, Fatemi R. The effect of exercise on learning and spatial memory following stress-induced sleep deprivation (sleep REM) in rats. Jentashapir J Health Res. 2016;7(2):1-8. doi: $10.17795 /$ jjhr-32005.

26. Berchtold NC, Castello N, Cotman CW. Exercise and time-dependent benefits to learning and memory. Neuroscience. 2010;167(3):58897. doi: 10.1016/j.neuroscience.2010.02.050. [PubMed: 20219647]. [PubMed Central: PMC2857396].

27. Fernandes J, Arida RM, Gomez-Pinilla F. Physical exercise as an epigenetic modulator of brain plasticity and cognition. Neurosci Biobehav Rev. 2017;80:443-56. doi: 10.1016/j.neubiorev.2017.06.012. [PubMed: 28666827]. [PubMed Central: PMC5705447].

28. Reger MA, Watson GS, Green PS, Baker LD, Cholerton B, Fishel MA, et al. Intranasal insulin administration dose-dependently modulates verbal memory and plasma amyloid-beta in memory-impaired older adults. J Alzheimers Dis. 2008;13(3):323-31. doi:10.3233/JAD-2008-13309. [PubMed: 18430999]. [PubMed Central: PMC2804944]

29. Bitgul G, Tekmen I, Keles D, Oktay G. Protective effects of resveratrol against chronic immobilization stress on testis. ISRN Urol. 2013;2013:278720. doi: 10.1155/2013/278720. [PubMed: 24307953]. [PubMed Central: PMC3836419].

30. Duarte AI, Proenca T, Oliveira CR, Santos MS, Rego AC. Insulin restores metabolic function in cultured cortical neurons subjected to oxidative stress. Diabetes. 2006;55(10):2863-70. doi: 10.2337/db06-0030. [PubMed: 17003354].

31. Muller AP, Gnoatto J, Moreira JD, Zimmer ER, Haas CB, Lulhier F, et al. Exercise increases insulin signaling in the hippocampus: Physiological effects and pharmacological impact of intracerebroventricular insulin administration in mice. Hippocampus. 2011;21(10):1082-92. doi: 10.1002/hipo.20822. [PubMed: 20824731].

32. Murray DK, Sacheli MA, Eng JJ, Stoessl AJ. The effects of exercise on cognition in Parkinson's disease: A systematic review. Transl Neurodegener. 2014;3(1):5. doi: 10.1186/2047-9158-3-5. [PubMed: 24559472]. [PubMed Central: PMC3936925] 